\title{
Geodetic monitoring of deflections of reinforced beams and reinforcing elements under variable static loading
}

\author{
Igor Rubtsov ${ }^{1, *}$ and Oleg Rubtsov ${ }^{1,2}$ \\ ${ }^{1}$ Moscow State University of Civil Engineering, 26, Yaroslavskoye Shosse, 109377, Moscow, Russia \\ ${ }^{2}$ Higher School of Economics, 10, Myasnitskaya str., 102125, Moscow, Russia
}

\begin{abstract}
In the framework of the present study was carried out monitoring of reinforced concrete structures subjected to reinforcement. As reinforcement elements, steel channels were used, rigidly connected to a reinforced concrete beam to be reinforced. Object of research: systems of quasi-continuous observations of the parameters of the construction object carried out on the basis of the monitoring system. Subject of research: identification of the possibility of using various systems, in particular, geodetic and strain gauge monitoring, to monitor the bending of reinforced concrete elements and, in particular, reinforced concrete beams. Aim: Comparative analysis of strain gauge and geodetic monitoring in the process of fixed changes in static loads on structural elements. Materials and methods: the initial materials for this study were the results of geodesic and strain gauge monitoring during statistical step-by-step loading of reinforced concrete beam by steel channels. Results: on the basis of the obtained dependences of the deflection of elements of reinforced and reinforcing structures in time during their step-by-step statistical loading the possibility of using geodetic control is shown. Conclusions: The possibility of using geodesic and strain gauge methods for monitoring reinforced concrete structures is proved.
\end{abstract}

\section{Introduction}

During the research, prismatic reflectors were installed on the existing beam from the moment of construction of the building and reinforced during reconstruction works for geodetic measurements of the movements of the observed zones of the beam when it is loaded. Observations were made using a robotic total station that provides a measurement period of several minutes.

During the monitoring process, monitoring was carried out both for prisms located on the reinforced element-a reinforced concrete beam, and for prisms installed on reinforcing elements-channels installed on both sides of the reinforced concrete beam. Based on a comparison of the movements of the observed elements - prisms installed on the beam and channels, an assessment of their compatibility can be made. In the end, it can be concluded

\footnotetext{
*Corresponding author: massalski56@gmail.com
} 
about the quality of the reinforcement, the reliability of the joint operation of the amplified and reinforcing elements, and the possibility of subsequent operation.

\section{Literature review}

One of the acknowledged methods so far to ensure the safety of building structures is to monitor the technical condition of these structures. Monitoring can and should be carried out at the stage of construction of the structure [1] and at the stage of its operation [2]. The legal basis for monitoring is the regulatory and technical framework. Brief information about these frameworks is given in [3-5]. Probabilistic and statistical methods for processing the results of monitoring and its implementation are described in paper [6].

Monitoring of building structures can be carried out in various ways and methods. These, in particular, include geodetic methods [7], [28], including methods of geodetic monitoring of architectural monuments that have a long service life [8]. Monitoring based on non-destructive concrete testing methods is described in papers [10], [25]. The age of the concrete of the surveyed structures is also important [11]. Monitoring of the stressstrain state of structural elements can be carried out on the basis of measurement of relative deformations by electric or fiber-optic strain gauges [12-13], [29-30] or other methods and ways. The influence of ambient temperature and associated with it deformations during geodetic monitoring is described in [14].

The literature provides a large amount of information related to the monitoring of building structures, including various types of tests of these structures. The load-bearing capacity of a building structure can be determined, in particular, under their static tests. In such tests, their deformations are monitored under the influence of a controlled load, which contributes to the second group of limit states. As a result of early researches, the deviation from the original position of some structural elements of structures is given in the works [15-18]. In particular, the results of the study of reinforced beams are given in [19-26], and the results of tests of t-beams are given [27]. Vibrations have a specific effect on structures, as described in [28].

\section{Materials and methods}

The tested structure is a three-span reinforced concrete beam with a t-section. The spans of the beam in the light were 5800, 3000 and $5800 \mathrm{~mm}$. The edges of the beam are supported by wall structures. The central part of the beam is supported by column. The size of the column on the scheme is $700 \times 700 \mathrm{~mm}$. During the reconstruction work, the beams were reinforced with channels No 24, located symmetrically to the axis of the brand on both sides. A schematic cross section of the beam is shown in figure 1.

The upper part of the beam has a monolithic reinforced concrete floor, which is also shown in figure 1 . The floor beams in the studied part of the building are arranged in increments of $3000 \mathrm{~mm}$.

In the process of experimental studies, a load was made on one of the spans of the beam with a length of $5800 \mathrm{~mm}$. The load was carried out by loading the span of the beam by placing sandbags on the floor of the floor located above the beam. All bags had the same weight, which made it easier to count the load. The bags were placed on a pre-defined strip of floor, symmetrically located above the beam. The width of the strip is $3000 \mathrm{~mm}$. During the experiment, a gradual, uniform loading of the beam was carried out. The load at the first stage was $48 \mathrm{~kg} / \mathrm{m}^{2}$. At this load, a 10-minute delay was performed prior to the changes. After the measurements, the load was increased by $48 \mathrm{~kg} / \mathrm{m}^{2}$. Thus, in the second stage, it was $96 \mathrm{~kg} / \mathrm{m}^{2}$. After 10 minutes of exposure and measurements, another laying of sandbags 
was carried out. The load reached $144 \mathrm{~kg} / \mathrm{m}^{2}$. In the fourth stage, the evenly distributed load was increased to $192 \mathrm{~kg} / \mathrm{m}^{2}$. At the last fifth stage, it was $240 \mathrm{~kg} / \mathrm{m}^{2}$. At the same time, the shutter speed preceding the measurements was increased to 30 minutes. After the measurements of the monitored parameters has undergone gradual unloading of the beams. Unloading was performed in the same sequence as loading. The exposure time after removing the load was also 10 minutes before the measurements.

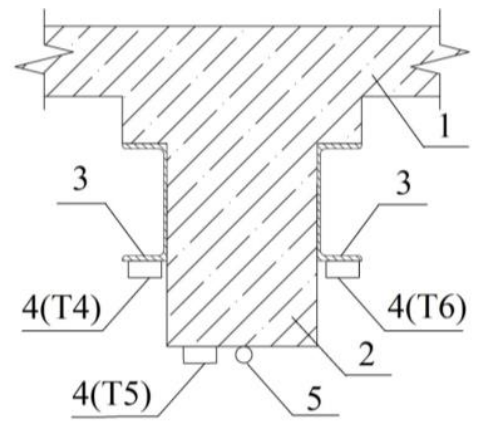

Fig. 1. Cross-section diagram of the investigated structure, where: 1-floor structure; 2-t-shaped threespan continuous reinforced concrete beam; 3-channel; 4 - location of GMP-104 mini-prisms with $L$ shaped holder; 5 - location of the strain gauge.

Deformations of the loaded beam were determined by geodetic methods using GMP104 prism reflectors installed in the middle of the beam span. The reflectors were mounted on L-shaped holders mounted on two channels and on a reinforced concrete beam. The diagram of the prism mounting locations is shown in figure 1. The Leica TCR 1200 series robotic total station was used to measure the deflection of the beam. The total station program provided for fixing the value of the strain on three coordinates with equal time intervals throughout the measurements. In total, 100 cycles of three-coordinate survey were performed using three prisms.

\section{Results of the research}

As noted above, during the study, a step-by-step loading of the beam was performed while fixing its deflection at three points located in the middle of the span. Two of them are fixed on the reinforcement elements-channels and one on the reinforced element - on the surface of the reinforced concrete beam. The dependences of the beam deflection, expressed in vertical movements of the observed points, on the applied load is reflected in the presented figures. So, the graphs presented in figure 2 and figure 4 show the vertical movements of the T4 and T6 prisms installed on the channel. The graph in figure 3 shows the vertical movement of the prism fixed directly to the reinforced element-a reinforced concrete beam. The vertical positions of the observed points at the time of measurement are plotted along the ordinate axis. Along the abscissa axis, time counts corresponding to the measurement moments are deferred.

In addition to the actual value of the measured parameters, indicated on the graph by green points, the graphs show lines of their average values of red points and lines corresponding to a possible measurement error, i.e., tolerance, marked by blue line. [27].

In addition, the loading diagram is conditionally shown from the graphs by a polyline 


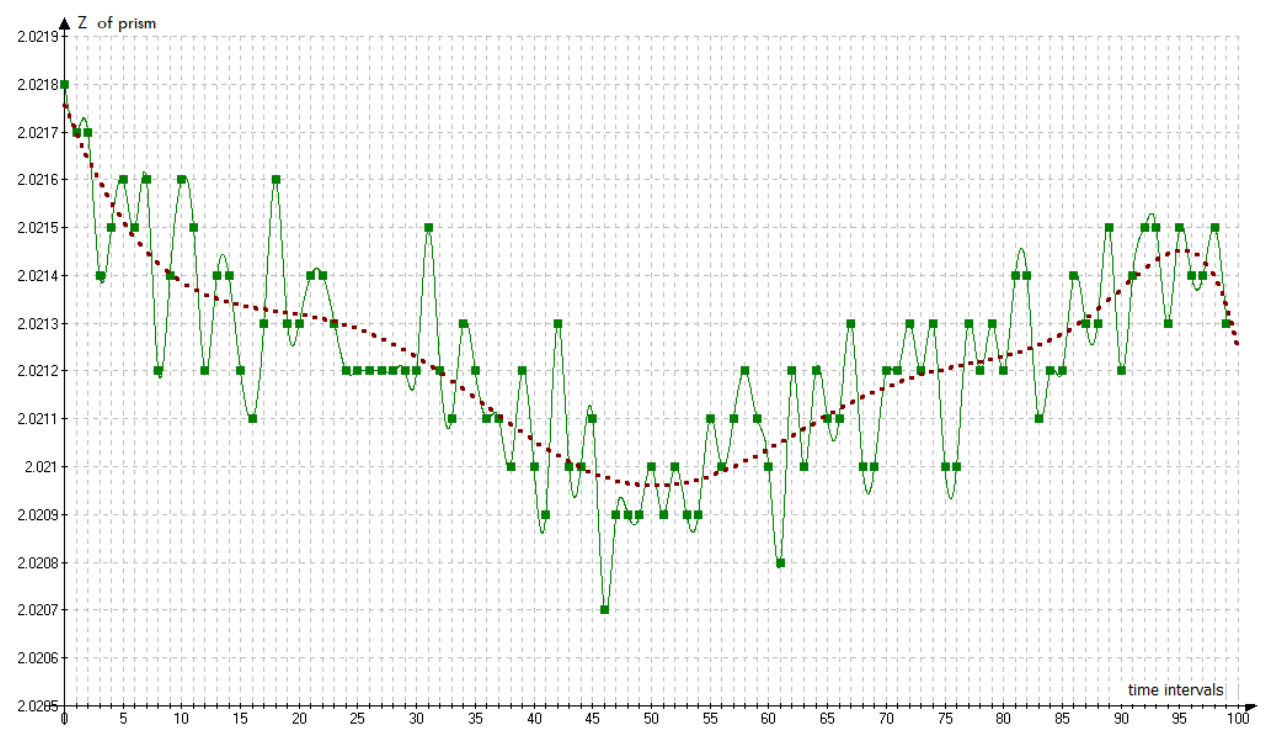

Fig. 2. Vertical displacements of the T4 prism mounted on the channel.

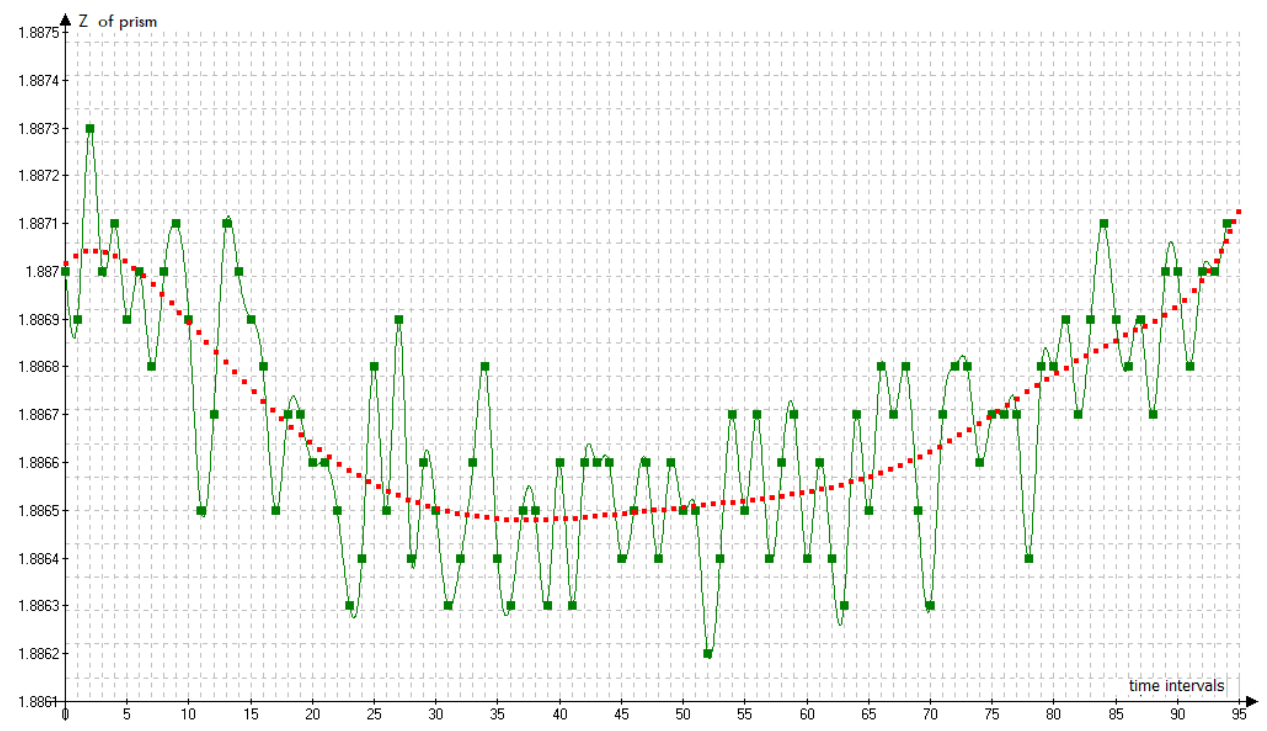

Fig. 3. Vertical displacements of the T5 prism mounted on concrete. 


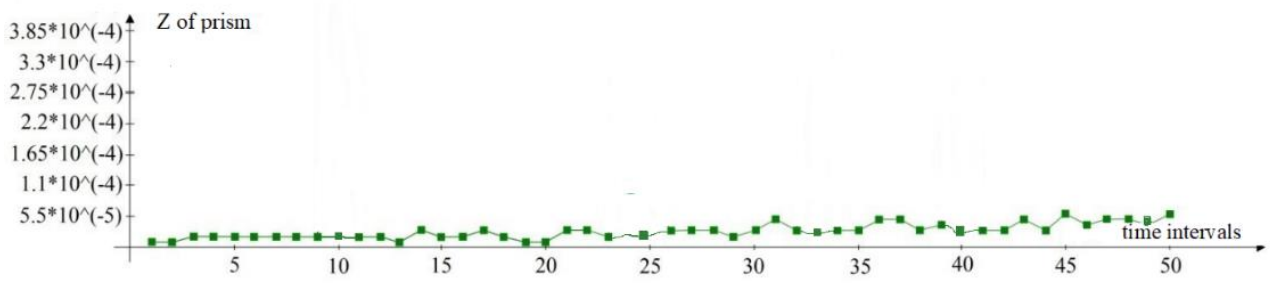

Fig. 4. Vertical displacements of the T6 prism mounted on the channel.

Consider the graphs shown in figures 2-4. At the same time, once again we pay attention to the fact that the graphs shown in figures 2 and 4 show the deformation of reinforcing elements, i.e. channels. The graph in figure 3 shows the deformation of the reinforced element itself - a reinforced concrete beam. First, we see a well-viewed dependence of the strain on the load value. The load value is represented by a broken line at the bottom of each graph. Secondly, the proximity of the maximum values of deflection values, namely the deflection value: for the T4 prism installed on the channel, the maximum deformation is $0.85 \mathrm{~mm}$; for the $\mathrm{T} 6$ prism also installed on the channel, the maximum deformation was the same $0.85 \mathrm{~mm}$; for the $\mathrm{T} 5$ prism installed on a reinforced concrete beam, the maximum deformation was $0.7 \mathrm{~mm}$.

Comparison of deflections shows the correctness of choosing a design solution for strengthening the beam, which consists in the fact that most of the load is taken by the force elements - their deflection is $0.85 \mathrm{~mm}$. the strength of the reinforced beam is slightly less $0.7 \mathrm{~mm}$.

The deformations of the elements under consideration are in the submillimeter range. How justified is the use of a total station during measuring? Let's examine at this question in more detail.

The information about the standard deviation for single measurements at a distance from 1950 to $50159 \mathrm{~mm}$ is provided in the gold certificate (Leica Geosystems Calibration Certificate). The standard deviation is $0.21 \pm 0.08 \mathrm{~mm}$. In this case, $0.21 \mathrm{~mm}$ is a system error and $0.08 \mathrm{~mm}$ is a random error. In the case of continuous observations of the deflection of the beam from a single installation point, the system error does not affect the result. Note that the calibration protocol of the measuring instrument - in our case, the total station-indicates the measurement error relative to the reference basis, which is $0.04 \div 0.05$ $\mathrm{mm}$. Also, the protocol specifies the extended measurement uncertainty - the spread of values when repeatedly pointing at the measured point in accordance with the protocol ranges from 0.04 to $0.05 \mathrm{~mm}$. The extended uncertainty is essentially a variance- $\sigma$. At a $95 \%$ confidence interval, the spread of values is defined as $2 \sigma$, i.e. from 0.08 to $0.10 \mathrm{~mm}$.

So, based on the certificate, we can say that the measurement error during the described observations did not exceed $0.1 \mathrm{~mm}$, which is reflected in the graph of figures 2-4.

Analysis of the results of the experiment showed that the deviations in the values of the measured deformations do not exceed $0.06 \mathrm{~mm}$. In order to confirm these values, a laboratory experiment was conducted. Its essence was that the rigidly fixed total station Leica TCR1200 sequentially, (in automatic mode), was guided for a long time on two prisms. These actions were performed under conditions that are closest to the conditions of the experiment described above for determining the deflection of beams. The main difference was that the load on the beam did not change during the experiment. The analysis of the results of the experiment shown that the deviations in the values of the measured deformations do not exceed $0.06 \mathrm{~mm}$. This data is well correlated with the above values of the extended uncertainty, namely 0.04-0.05 $\mathrm{mm}$. 


\section{Conclusions}

The results obtained confirm the hypothesis of the necessity and sufficiency of geodetic monitoring when observing the state of building structures, even with their minor deformations.

The observed points in the study of reinforced structures should be located both on the reinforced and on the reinforcing elements.

Geodetic monitoring allows identifying the degree of compatibility of the amplified and reinforcing elements.

It is proved that high-precision total stations can be used for monitoring structural deformations, including during loading.

Monitoring carried out with the help of robotic total stations allows tracking the deformation of the structure over time, and therefore take into account the accumulation of damage in the structure during its periodic loading, including alternating loading.

\section{References}

1. I.V. Rubtsov, Integral 5, 86-87 (2007)

2. I.V. Rubtsov, Integral 6, 102-103 (2007)

3. A.V. Korgin, M.A. Zakharchenko, M.V. Emelianov, V.A. Ermakov, I.V. Rubtsov, A.V. Kukhta, Vestnik of Moscow State Construction University 8, 212-221 (2011)

4. N.P. Chetverik, I.V. Rubtsov, Occupational safety in industry 4, 38-40 (2008)

5. I.V. Rubtsov, N.P. Chetverik, Science and security. 1(10), 2-27 (2014)

6. O.I. Rubtsov, I.V. Rubtsov, Industrial and civil construction 6, 44-45 (2006)

7. I.V. Rubtsov, Theoretical Foundation of Civil Engineering Proceedings, 409-417 (2013)

8. I.V. Rubtsov, T.A. Pyatnitskaya, Vestnik of Moscow State Construction University 5, 80-86 (2013)

9. R.Y. Yakubovsky, I.A. Bulanov, I.A.Alper, N.A. Klepikova, A.A. Kazakov, Young scientist 23(103), 280-283 (2015)

10. K.R. Kashapova, V.I. Kleveko, Scientific works of the S Word 12.4 (41), 90-94 (2015)

11. L.N. Kolomiets, T.N.Murashkina, I.V. Rubtsov, Construction materials, equipment, technologies of the XXI century 3, 40-44 (2008)

12. L.N. Kolomiets, T.I. Murashkina, I.V. Rubtsov, Sensors and systems 2, 49-63 (2008)

13. I.V. Rubtsov, I.A. Nazarov, E.D. Lavrinenko, V.P. Savushkina, Vestnik of Moscow State Construction University 4-5, 329-334 (2010)

14. A.A. Gassiev, T.V. Morozova, A.V. Gornostaev, I.M. Mogushkov, Safety of structures 1, 87-91 (2011)

15. D. Khodzhaev, B. Normuminov, Y. Mustapakulov, A.Mottaeva, E3S Web of Conferences, 110, 01045, (2019) doi.org/10.1051/e3sconf /201911001045

16. V.N. Migunov, Online journal of the sociology of Science 5(24), 5 (2014)

17. S.G. Parfenov, A.A. Pronin, Y.D. Vinokurov, I.A. Rubtsov, Youth and the XXI century-2016, Materials of the VI international youth scientific conference, 281-283 (2016)

18. V.O. Bulavenko, I.S. Guchkin, Regional architecture and construction 2, 117-122 (2014) 
19. S.V. Myamlin, O.H. Reidemeister, A.L. Pulariia, V.O. Kalashnyk, Science is progressing for transport 6(60), 118-126 (2015)

20. S.V. Myamlin, O.H. Reidemeister, A.L. Pulariia, V.O. Kalashnyk, Science is the progress to transport 5(59), 132-140 (2015)

21. N.V. Frolov, M.A. Poloz, E.G. Kolesnikova, Bulletin of the Belgorod state technological University 10, 83-88 (2016)

22. F.S. Zamaliev, V.V. Filippov, Industrial and civil construction 7, 29-36 (2015)

23. M.P. Koval, Science and the World 1.6(22), $42-47$ (2015)

24. P.V. Ysniy, O.M. Yakovishin, D.I. Dubianskii, Modern technologies, materials and structures in construction 2(13), 20-23 (2012)

25. A.A. Bykov, V.N. Snigireva, A.A. Koryagin, Bulletin of the Perm national research Polytechnic University. Applied ecology. Urbanistics 3(23), 147-159 (2016)

26. F.S. Zamaliev, E.G. Bikkinin, Proceedings of the Kazan state University of architecture and construction 4, 134-138 (2014)

27. I.V. Rubtsov, A.M. Galushko, E.D. Lavrinenko, Scientific and technical Bulletin of the Volga region 6, 363-365 (2012)

28. I. Rubtsov, R. Alisultanov, A. Zinatullin, N. Midrigan, MATEC Web of Conferences (2018)

29. O. Rubtsov, R. Alisultanov, N. Rogova, E3S Web of Conferences, 03027 (2019)

30. R.S. Alisultanov, A.V. Oleynikov, M.V. Sryvkova, M.Y. Proshin, Vestnik Moscow State Construction University 10, 7-19 (2015) 